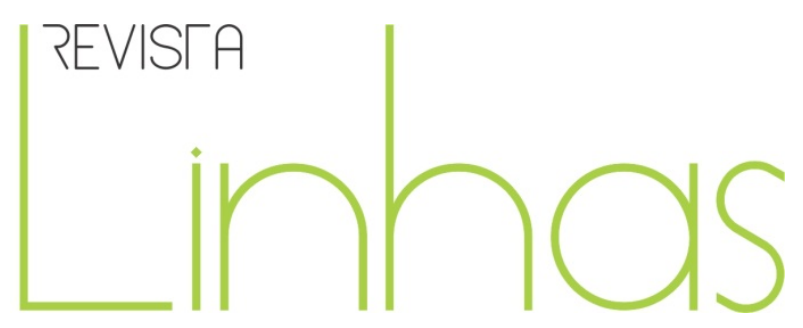

\title{
Un essai de démocratisation universitaire en France: histoire de l'université de Paris VIII Vincennes (1968-1980) ${ }^{1}$
}

\begin{abstract}
Résumé
Dans les années 1970 l'université de Vincennes, dont le département de sociologie est fondé par Jean-Claude Passeron et celui de philosophie par Michel Foucault, est le lieu d'une tentative originale de démocratisation de l'université française. Après avoir décrit ses circonstances de création et son public, nous tirerons un bilan pédagogique de cette expérience pédagogique hors du commun. Sachant que cette université accueillera en masse des étudiants non bacheliers, ainsi qu'une très forte proportion d'étudiants salariés. Un bilan quelque peu mitigé attendu que sous la pression politique du moment, du désordre ambiant, comme d'oppositions de classes larvées, les enseignants et les étudiants n'arriveront guère à s'accorder sur un minimum de principes pédagogiques communs. Et un des résultats pour le moins paradoxal de cette expérience est que les vincennois inventeront une sorte de marché libre académique où régnera la concurrence charismatique entre tous les enseignants.
\end{abstract}

Mots-clés: Université de Vincennes; Démocratisation; Pédagogie; Jean-Claude Passeron; Discipline.

\section{Para citar este artigo:}

SOULIÉ, Charles. Un essai de démocratisation universitaire en France: histoire de l'université de Paris VIII Vincennes (1968-1980). Revista Linhas. Florianópolis, v. 15, n. 29, p. 13-41, jul./dez. 2014.

DOI: $10.5965 / 1984723815292014013$

http://dx.doi.org/10.5965/1984723815292014013

\footnotetext{
${ }^{1}$ Cet article se fonde sur un ouvrage collectif intitulé Un mythe à détruire? Origines et destin du Centre universitaire expérimental de Vincennes, dir. Charles Soulié, Presses universitaires de Vincennes, 2012.
} 


\section{An attempt to university democratization in France: the history of the University of Paris VIII Vincennes (1968-1980)}

\begin{abstract}
During the 1970s, the University of Vincennes - whose Department of Sociology was founded by Jean-Claude Passeron, its Department of Philosophy by Michel Foucault - was the site of a novel project to democratize the French university. After describing the University's circumstances of creation and its public, we will sum up the pedagogical lessons of this unusual educational experiment. It was a university that welcomed masses of students without a baccalauréat (secondary school diploma), along with a very large proportion of working students. Yet our evaluation must be tempered by the fact that - given the political pressures of the times, the disorderly atmosphere, the latent class conflicts - its teachers and students never really managed to agree on a minimum set of shared pedagogical principles. And one of the rather paradoxical results of this experiment was that Vincennes would invent a sort of academic free market, ruled by charismatic competition between all its teachers.
\end{abstract}

Keywords: Université de Vincennes; Democratization; Pedagogy; Jean-Claude Passeron; Disciplines.

Uma tentativa de
democratização universitária
na França: história da
universidade de Paris VIII
Vincennes (1968-1980)

\section{Resumo}

Nos anos 1970, a Universidade de Vincennes, cujo departamento de sociologia foi fundado por Jean-Claude Passeron e o de filosofia por Michel Foucault, é o lugar de uma tentativa original de democratização da universidade francesa. Após descrevermos as circunstâncias de criação e o seu público, faremos um balanço pedagógico desta experiência pedagógica fora do comum, sabendo que esta universidade acolheu em massa estudantes sem bacharelado, assim como uma grande proporção de estudantes assalariados. Um balanço um tanto modesto, uma vez que, sob a pressão política do momento, da desordem instaurada, assim como das oposições entre classes latentes, os professores e os estudantes não conseguiram entrar em acordo sobre princípios pedagógicos comuns mínimos. E um dos resultados no mínimo paradoxal dessa experiência é que os vincennois inventaram uma espécie de mercado livre acadêmico no qual reinou a concorrência carismática entre todos os professores.

Palavras-chave: Universidade de Vincennes; Democratização; Pedagogia; Jean-Claude Passeron; Disciplina. 
Quand, en 1964, Pierre Bourdieu et Jean-Claude Passeron font paraître Les Héritiers, l'université française connaît une véritable crise de croissance. Ainsi entre 1945 et 1960 , le nombre d'étudiants passe de 123.000 à 214.000 . Mais cette croissance s'emballe à partir des années 1960, le taux de progression annuel étant généralement supérieur à 10\%. Ainsi à la rentrée 1967, on compte 504.540 étudiants.

Cette expansion très rapide s'accompagne d'un changement de la composition scolaire, sociale du public étudiant. Et qui n'est pas sans susciter des réactions malthusiennes dans le corps enseignant. Ainsi au début des années 1960, «on voit le ton monter ou s'aigrir, dans les reportages de presse qui découvrent la "grande misère des universités" comme dans les "prises de parole en amphi”, qui déjà se "surpeuplent" ou dans les apartés sentencieux des professeurs qui ne reconnaissent plus dans leurs étudiants les mimétismes de disciples ou de successeurs: "le niveau des étudiants baisserait-il" d'année en année plus vite encore que ne s'y étaient depuis toujours résignés les professeurs qui aiment à répéter, d'année en année, cette phrase de mémoire perdue ? " ${ }^{2}$

Cette massification conduit le gouvernement à recruter nombre de nouveaux enseignants. Ainsi entre 1959 et 1967, le nombre de professeurs, maîtres de conférences, maîtres assistants et assistants en lettres et sciences humaines passe de 974 à 3.908. Ce qui s'accompagne aussi d'un changement de la structure des emplois, les effectifs d'enseignants subalternes croissant beaucoup plus rapidement que ceux de professeurs. Ce qui limite alors considérablement les possibilités de carrière et aura des répercussions politiques importantes en mai $68^{3}$.

Mais elle conduit aussi à la création de nouveaux établissements universitaires publics, déjà en province. Ainsi en France en 1968, on compte 40 villes universitaires contre 24 en $1939^{4}$. Et cette croissance concerne aussi la région parisienne, beaucoup d'articles de presse soulignant le fait que "la Sorbonne étouffe dans ses murs». L'université d'Orsay, centrée sur les sciences, ouvre donc ses portes en 1958. Et celle de

\footnotetext{
2 Cf. Jean-Claude Passeron, "1950-1980: l'université mise à la question: changement de décor ou changement de cap ? ", dans Jacques Verger (dir.), Histoire des universités en France, Toulouse, Privat, 1986, p. 368.

${ }^{3}$ Sur ce point, voir Pierre Bourdieu, Homo academicus, Minuit, 1984. Et plus spécialement le chapitre intitulé « Le moment critique ».

${ }^{4}$ Antoine Prost, Histoire générale de l'éducation, Paris, Perrin, vol. IV, 2004, p. 313.
} 
Nanterre, «fille de la Sorbonne », consacrée aux lettres et sciences humaines ainsi qu'au droit et à l'économie, et qui, avec son département de sociologie où enseignaient notamment Alain Touraine, Michel Crozier, Henri Mendras, Henri Lefebvre, etc., sera un des épicentres de la révolte universitaire de mai 68, est inaugurée en 1964.

De même à l'automne 68, et en réponse aux critiques adressées en mai 68 à l'université traditionnelle (élitisme, immobilisme intellectuel, absence de démocratie interne, etc.) le pouvoir gaulliste fait voter une Loi d'orientation transformant profondément le statut des universités françaises. Celles-ci acquièrent alors une première forme d'autonomie et développent la «participation" (c'est-à-dire des formes de démocratie interne), ainsi que la pluridisciplinarité. Et il décide d’ouvrir en toute urgence à Paris deux Centres universitaires expérimentaux aux destinées bien divergentes: Dauphine, consacrée aux sciences du management et implantée dans les «beaux quartiers » de la capitale, et Vincennes quelque peu isolée car construite au cœur d'un grand bois parisien, en l'occurrence le Bois de Vincennes et centrée sur les lettres et sciences humaines.

Dans le cadre de cet article, nous nous intéresserons au destin de l'université de Vincennes, dont un des objectifs principaux sera notamment de démocratiser l'accès aux études universitaires. Université dans laquelle s'engagera notamment Jean-Claude Passeron, futur directeur de son département de sociologie et qui, dans la lignée de ses travaux avec Pierre Bourdieu, pense pouvoir y trouver un lieu d'expérimentation d'une pédagogie plus rationnelle ${ }^{5}$. Nous décrirons déjà les circonstances de création de cette université hors du commun, son public, pour tirer ensuite un bilan pédagogique de cette expérience.

\footnotetext{
5 Rappelons que lorsqu'en 1970 Pierre Bourdieu et Jean-Claude Passeron publient La Reproduction, Passeron enseigne à Vincennes.
} 


\section{Une création sous haute tension politique}

En ouvrant les centres universitaires expérimentaux de Dauphine comme de Vincennes, le pouvoir gaulliste poursuit un double objectif. D'une part montrer qu'il a entendu les critiques énoncées en mai et permettre aux novateurs de lancer des expérimentations pédagogiques et scientifiques susceptibles ensuite, si elles aboutissent, d'irriguer l'ensemble de l'enseignement supérieur. Mais aussi éloigner du centre de Paris, et plus précisément du Quartier latin, ses éléments les plus perturbateurs, de façon notamment à faciliter le « retour à l'ordre ». Et de fait à l'époque, et suite aux évènements de mai 68, une partie de la jeunesse étudiante croit à l'imminence d'une prochaine révolution et nombre d'universités sont d'intenses foyers de contestation politique. Les mouvements politiques d'extrême gauche, et notamment ceux d'inspiration maoïste, sont en plein essor et marxisme et pensée critique se diffusent largement, tant chez les étudiants que chez les enseignants.

Edgar Faure est alors ministre de l'Éducation nationale. C'est un politicien habile et qui manifestement a le sens de l'histoire. Ainsi à l'automne 1968 et lors du vote de la Loi d'orientation au sénat, il déclare : «Il n'y a pas d'exemple dans l'Histoire qu'une révolution ait été faite simplement par des révolutionnaires. Il faut donc que ces révolutionnaires entraînent avec eux des réformistes : mais nous faisons tout ce qu'il faut pour qu'ils n'y parviennent pas. " ${ }^{6}$ L'ouverture de ces deux centres universitaires expérimentaux, comme les recrutements massifs d'enseignants chercheurs après 1968, sont quelques-uns des éléments clef de la stratégie gouvernementale de l'époque. Un chiffre donne une idée de l'ampleur des largesses budgétaires en la matière. En 1967 on compte 3.908 enseignants chercheurs en lettres et sciences humaines. En 1974, ils sont 8.011. Soit un doublement du corps en sept ans. Alors que dans la même période, les effectifs d'étudiants en lettres et sciences humaines passent de 157.000 à 248.000 .

On a donc beaucoup recruté après 1968 et souvent dans des conditions peu orthodoxes. Ce qui s'explique notamment par la destruction du pouvoir mandarinal à l'ancienne, et donc du pouvoir gérontocratique, et qui débouche notamment sur une

\footnotetext{
${ }^{6}$ Philosophie d'une réforme, Paris, Plon, 1969, p. 108-109.
} 
période d'interrègne prolongée propice aux expérimentations, comme aux dérives en tout genre. Et ces recrutements massifs - qui s'arrêtent net après 1974 pour ne reprendre qu'à la fin des années 1980 à la faveur de la seconde massification universitaire - seront à l'origine de générations universitaires fortement différenciées. Ce qui aura des effets de long terme tant sur l'histoire institutionnelle de l'université française, que sur son histoire intellectuelle.

Alors à ses débuts, Vincennes est essentiellement un rêve d'enseignants et ceux-ci - par peur de se voir débordés par les étudiants les plus radicaux - les tiendront à distance le plus longtemps possible. Mais ces enseignants ne forment pas un groupe homogène et globalement on peut les diviser en deux groupes. Ou plutôt trois, si on ajoute la foule de ceux qui verront d'abord dans Vincennes une opportunité pour faire carrière, ou remonter plus vite à la capitale. En effet, et en raison de la centralisation extrême du système scolaire, universitaire, mais aussi de recherche, éditorial, etc., français, comme de la concentration de toutes les formes de capitaux sur Paris, une carrière académique réussie devait alors nécessairement se conclure à la capitale et plus exactement à la Sorbonne, ou au Collège de France.

Le premier groupe d'enseignants, le plus visible médiatiquement et sur lequel épiloguera d'ailleurs longuement l'hebdomadaire de la gauche intellectuelle de l'époque, en l'occurrence le Nouvel Observateur, est constitué par l'avant-garde intellectuelle française (et donc parisienne...) des années 60 et il est surtout préoccupé par des questions de recherche. Se retrouvent donc à Vincennes des novateurs en tout genre peinant à trouver leur place dans l'université traditionnelle. Et qui, en mai 68, rejoignent les étudiants dans leur critique de l'université, comme de la société en général. Nous décrirons ici plus précisément ceux des départements de philosophie et de sociologie, pour lesquels nous disposons de plus d'informations et qui joueront un rôle clef dans notre propos.

Ainsi en philosophie on peut citer les noms de Michel Foucault, Alain Badiou, Jacques Rancière, François Châtelet, Michel Serres, puis Gilles Deleuze, Jean-François Lyotard, etc. L'investissement de Michel Foucault dans l'expérience vincennoise fait grand bruit. Et c'est lui qui recrute les premiers enseignants du département de 
philosophie, dont il sera d'ailleurs le premier directeur. Alors en raison de la conjoncture politique du moment, ces recrutements s'opèrent sur des bases tant politiques que scientifiques. En effet, Foucault souhaite au départ créer "un centre d'analyse de la science dans sa dimension politique ». Lequel articulerait donc épistémologie et analyse politique. Mais quand il arrive à Vincennes, Foucault vient juste de Tunisie. Il n'a donc pas, comme on dit à l'époque, «fait 68 ». C'est-à-dire qu'il n’a pas été « sur les barricades », dans les commissions de réflexion de la Sorbonne, etc. Et donc, il connait mal le milieu des jeunes philosophes engagés. Ainsi pour le recrutement, il fait appel à Alain Badiou.

A l'époque Badiou, un normalien fils de normaliens élève d'Althusser et de Canguilhem et dont le père, enseignant en sciences, avait été maire de la grande ville de Toulouse, a 31 ans. C'est un militant maoïste très actif et très efficace en assemblée générale... Et il conseille Foucault pour les recrutements dans la jeune génération des normaliens philosophes élèves d'Althusser et de Lacan. Manifestement, les critères politiques jouent un rôle central dans ces recrutements. Et afin de contrebalancer l'influence maoïste très majoritaire, Foucault recrute Henri Weber, un dirigeant trotskyste, ainsi qu'Etienne Balibar, membre du Parti communiste français. Enfin, et pour jouer un rôle de modérateur dans ce milieu très militant, il fait appel à François Châtelet.

Du côté des sociologues, on relève notamment les noms de Jean-Claude Passeron, Robert Castel, Emmanuel Terray, Nicos Poulantzas, etc. C'est donc Jean-Claude Passeron, 38 ans à l'époque et maître de conférences à l'université de Nantes, dont le père était employé et la mère institutrice, qui est à l'origine du département de sociologie de Vincennes. Et c'est lui qui, avec entre autres le concours de Michel Foucault son aîné qu'il avait connu, et admiré, alors qu'il était élève à l'Ecole normale supérieure, recrute ses premiers enseignants, notamment en puisant du côté des althussériens ${ }^{7}$. Pierre Bourdieu est aussi sollicité, mais décline l'offre. Voici ce qu'en dit Jean-Claude Passeron lors d'un entretien : "Aron disait: "C'est très dangereux" et même Bourdieu, qui d'ailleurs a décliné. [...] Bourdieu a très vite compris - du moins ça l'amusait pas du tout -. Il m'a dit : "Non, mais

\footnotetext{
7 Signalons qu'en 1966, Jean-Claude Passeron avait aussi publié avec Gérald Antoine, un grammairien linguiste futur membre du cabinet d'Edgar Faure, un ouvrage intitulé La Réforme de l'université. Lequel insistait notamment sur "la naïveté sociologique des technocrates» et contient nombre d'extraits forts intéressants sur la pédagogie universitaire de l'époque, les équivoques de la démocratisation, etc.
} 
t'es complètement fou d'aller ramer sur cette galère". Effectivement, il était déjà en campagne pour le Collège de France. »

Quand il se lance dans l'aventure vincennoise, Jean-Claude Passeron peut notamment compter sur Robert Castel avec lequel, en 1967, il avait publié Éducation, développement et démocratie ${ }^{8}$. D’origine très populaire et provinciale (son père était un petit employé des ponts et chaussées et dans sa famille il n'y avait que deux livres), Robert Castel enseigne alors à la Sorbonne, où il vit donc les événements de 1968 : "j'étais à la Sorbonne, j'aurais pu y rester. Mais quand il y a eu cette opportunité de Vincennes, non que j'étais ultragauchiste et je ne pensais pas que l'on ferait la révolution, mais essayer de contribuer à une université plus ouverte, démocratique, moins traditionnelle... ${ }^{9}$

Le département de sociologie de Vincennes accueillant un nombre croissant d'étudiants, Jean-Claude Passeron et son équipe doivent ensuite recruter rapidement nombre d'enseignants de rang subalterne. Et ces derniers, généralement peu formés mais souvent très politisés à gauche, exerceront, avec le soutien des étudiants comme de la fraction la plus radicale des enseignants titulaires, une forte pression politique tant sur le fonctionnement de l'institution, les recrutements d'enseignants, que sur la constitution des programmes, modalités d'enseignement, etc.

A l'époque avant-garde intellectuelle et avant-garde politique semblent se rejoindre dans leur critique de l'ordre intellectuel, institutionnel, social et politique existant. Semblent, car les quiproquos, malentendus, phénomènes d'instrumentalisation réciproques, sont nombreux. Et de fait, légitimité intellectuelle et légitimité politique ne s'accordent pas nécessairement dans l'ambiance politiquement survoltée des facultés françaises de l'après 68 et les conflits entre générations universitaires sont parfois aigus. De même, les manifestations de terrorisme, tant intellectuel que politique, notamment portées par les groupes maoïstes et s'inspirant des pratiques de «la révolution culturelle » chinoise, seront légion.

\footnotetext{
${ }^{8}$ Paris, Mouton.

${ }^{9}$ Entretien avec Robert Castel le 6 décembre 2007.
} 
Le second groupe d'enseignants qui se lancera dans l'expérience vincennoise est constitué par des enseignants souvent moins connus, mais soucieux de rénover leur pratiques tant pédagogiques que scientifiques dans un sens à la fois plus démocratique et moderniste. Ce sont souvent, pour employer le vocabulaire politique de l'époque, des «réformistes ». C'est-à-dire des enseignants souhaitant profiter des dynamiques introduites en 68 par les étudiants à l'université pour renouveler les programmes, transformer le rapport pédagogique et ouvrir notamment l'université sur le monde, comme à de nouveaux publics.

Parmi eux, on peut penser aux enseignants des départements d'anglo-américain, ou de psychologie expérimentale, qui étaient alors de très gros départements et qui, pour cette raison d'ailleurs, formaient souvent quasiment à eux seuls une petite faculté avec tous les problèmes qu'entraine la gestion de grosses masses d'étudiants, comme d'enseignants $^{10}$. Ces enseignants, proches du Parti communiste français et souvent d'origine plus populaire que les enseignants d'avant-garde de renommée internationale, joueront un rôle clef au sens où ils assumeront notamment le rôle - quelque peu ingrat à l'époque - de la gestion quotidienne d'une institution hautement paradoxale, attendu qu'elle se voulait anti institutionnelle. Dans un univers symboliquement dominé par les valeurs critiques et libertaires notamment portées par les ténors intellectuels de l'époque, ils assumeront les positions de pouvoir local en participant notamment au fonctionnement de l'institution. Ce qui conduira parfois à des affrontements politiques violents.

Par exemple le 18 juin 1969, lors des premières élections tenues localement pour désigner les instances de gouvernement du Centre, les gauchistes et antiparticipationnistes balancent les urnes électorales par la fenêtre. En réaction, des enseignants du Centre rédigent un «Appel à tous les enseignants » signé par 150 d'entre eux et appelant à "la responsabilité de chaque enseignant»: "Devant ces événements, chaque enseignant est placé devant l'alternative suivante : ou bien il cautionne au nom d'une idéologie qui a décidé "la destruction de l’Université » ou par indifférence, les événements

\footnotetext{
${ }^{10}$ Concernant les origines, le recrutement et la politique du département d'anglo-américain de Vincennes, voir l'article de Marie-Pierre Pouly, «L'anglais de la Sorbonne à Vincennes », dans Un mythe à détruire?, op.cit., p. 281 et suivantes.
} 
de ce matin et, par là-même, la fermeture de Vincennes avec ses implications, ou bien il décide de prendre ses responsabilités et d'agir." En conséquence, les signataires s'engagent « à informer systématiquement dans leurs travaux pratiques leurs étudiants et à être personnellement présents le jour du vote pour assurer une libre consultation. " ${ }^{11}$ Tout ceci explique notamment l'ingouvernabilité chronique de cette université qui se prolongera jusqu'en 1971 environ et qui verra notamment une valse accélérée des doyens. Du reste, le phénomène n'est pas spécifique à Vincennes et s'observe aussi à Dauphine.

\section{Un public hors normes}

A l'origine, Vincennes peine à trouver ses premiers étudiants. Pourquoi ? Sans doute en raison du flou des objectifs d'un Centre à visée déjà expérimentale. Et de fait dans les années 1960, les facultés de lettres et sciences humaines françaises ont pour fonction essentielle de former des enseignants. Or le projet Vincennes qui veut s'ouvrir largement au monde contemporain, aux nouvelles disciplines, mais aussi aux emplois de l'avenir, semble vouloir rompre radicalement avec cette fonction traditionnelle. D'où sans doute la réticence à s'engager d'une frange des étudiants et notamment des plus intéressés par le professorat, qui sont aussi souvent les plus dotés scolairement et qui, pour cette raison, resteront dans les universités plus traditionnelles.

A l'origine, Vincennes attire essentiellement des étudiants de première année, dont beaucoup viennent de la Sorbonne ou de Nanterre et qui ont un profil qu'on pourrait qualifier d'héritiers critiques. Une partie d'entre eux sont donc très militants et souhaitent notamment faire de Vincennes «une base rouge » qui leur permettrait « d'abattre le capitalisme ». Mais il y a aussi nombre d'étudiants plus « réformistes », qui par exemple suivent leurs enseignants migrant de la Sorbonne à Vincennes. C'est par exemple le cas en histoire, ou en littérature française, départements politiquement moins

\footnotetext{
${ }^{11}$ Cf. Jean-Philippe Legois, « Jeux d'échelle entre les premiers pouvoirs universitaires vincennois, 1968-1971 », dans Un mythe à détruire?, op.cit., p. 266.
} 
radicaux que ceux de philosophie, sociologie ou arts plastiques. Mais on peut aussi parler des étudiants ordinaires ayant trouvé là une université particulièrement accueillante au plan des formalités d'inscription, exigences académiques, etc. Enfin dès la première année, on rencontre une proportion non négligeable d'étudiants non bacheliers (18\%). Ce qui nous conduit au cœur de notre objet.

En effet, la grande originalité de Vincennes résidera dans son public. Car dès son ouverture celle-ci s'ouvre résolument à une population habituellement rejetée de l'université : en l'occurrence celle des non bacheliers et des salariés. Grâce notamment à la multiplication des cours du soir, le samedi, à la très grande souplesse de ses cursus et aux facilités pour s'inscrire, Vincennes draine un public original. Ainsi en 1975 elle compte $38 \%$ de non bacheliers, $60 \%$ de salariés et 38\% de salariés à plein temps. En conséquence, la moyenne d'âge des Vincennois passe de 27 ans en 1973-1974, à 29 ans en 1978-1979, contre 22 ans dans les autres universités françaises.

Manifestement, cette université répond à une demande sociale forte. Initialement prévue pour 7.000 étudiants, elle en compte 32.000 en 1979 . Soit plus de quatre fois plus alors que les locaux, crédits, comme les recrutements d'enseignants et de personnels, ne suivent pas dans la même proportion. D’où, après une première période quasi luxueuse qui provoquera d'ailleurs la jalousie des autres universités, l'installation dans une misère matérielle chronique. Le ministère refusant aux autres universités le droit d'accueillir les non bacheliers et Vincennes acceptant tous ceux qui se présentent, très vite elle sera victime de son succès.

Enfin, dernière caractéristique du public vincennois, sa forte proportion d'étudiants étrangers. Ainsi en 1976, Vincennes compte $46 \%$ d'étudiants étrangers ${ }^{12}$. Et elle accueille aussi nombre d'enseignants étrangers, dont des réfugiés politiques ${ }^{13}$. Elle

\footnotetext{
${ }^{12}$ En 1977-1978, Vincennes accueille 14.871 étudiants étrangers, dont 1.677 américains. Parmi ces derniers on compte, par ordre décroissant : 297 colombiens, 262 brésiliens, 236 chiliens, 187 argentins, etc. Sur ce point : «Voyage en terre d'asile académique : éléments pour une histoire sociale des étudiants étrangers de Paris VIII et d'ailleurs ", in Un mythe à détruire?, p. 393 à 422.

${ }^{13}$ Par exemple après le coup d'Etat militaire de Pinochet au Chili, Vincennes accueille plusieurs enseignants chiliens dans ses départements d'histoire, comme de sociologie. Sachant aussi qu'au début des années 1970 le brésilien Josué de Castro enseigne à Vincennes. Ce qui sera aussi le cas de Mario Soares, futur président de la république du Portugal, et expulsé en 1970 de son pays par le gouvernement dictatorial de Marcello Caetano. Concernant ces deux enseignants: Alain Bué, "Josué de Castro, un visionnaire brésilien à
} 
est alors l'université la plus étrangère de France, ce qu'elle est encore aujourd'hui. Si l'on veut décrire structuralement le public de Vincennes, on peut dire qu'il est l'antithèse, à peu parfaite, du public particulièrement standardisé, et sélectionné, tant scolairement que socialement, des classes préparatoires et grandes écoles françaises. Classes, écoles qui, en France, concentrent donc une bonne part de l'élite scolaire du pays et d'où proviennent d'ailleurs la majorité des enseignants les plus renommés de Vincennes. Lesquels seront donc confrontés à un écart scolaire, social et culturel maximum dans leurs pratiques pédagogiques quotidiennes.

Pour reprendre le titre d'une affiche militante de l'époque on peut donc dire que, dans un bel élan de générosité collective plus ou moins utopique, Vincennes essaiera d'être une «Fac ouverte à tous", ou encore "aux travailleurs». Mais ce n'est pas parce qu'une institution ouvre formellement ses portes à tous, et donc que tout le monde rentre, que tout le monde tire un profit égal de son passage dans l'institution concernée $^{14}$. Par exemple, si on compare l'origine sociale des vincennois à celle des étudiants des autres universités, on observe que, finalement, elle en est très proche. Et que, comme dans les autres universités, le recrutement social s'élève à mesure qu'on monte dans le cursus.

\footnotetext{
Vincennes »: Mario Soares, «Exilé en cravate », in Jean-Michel Djian, Vincennes, une aventure de la pensée critique, Flammarion, 2009.

${ }^{14}$ Par analogie, on peut ici penser à ce qu'il en est de la fréquentation des musées. En effet, leur gratuité ponctuelle (que ce soit le dimanche ou un autre jour) ne suffit guère à assurer la démocratisation de leur public. En effet, celle-ci suppose l'acquisition préalable d'un certain capital culturel, comme de certaines dispositions cultivées. Sur ce point : L’Amour de l'art, Pierre Bourdieu et Alain Darbel, Minuit, 1966.
} 
Tableau n 1: L’origine sociale des étudiants en France et à Vincennes en 1975-1976

\begin{tabular}{|l|c|c|}
\hline & $\begin{array}{c}\text { France } \\
\text { (lettres et sciences } \\
\text { humaines) }\end{array}$ & $\begin{array}{c}\text { Vincennes } \\
\text { (ensemble des } \\
\text { disciplines) }\end{array}$ \\
\hline Profession libérale, cadre supérieur & $29,3 \%$ & $28,7 \%$ \\
\hline Cadre moyen & $17,8 \%$ & $18,7 \%$ \\
\hline Employé & $10,2 \%$ & $9,8 \%$ \\
\hline $\begin{array}{l}\text { Patron de l'industrie, du commerce, } \\
\text { agriculteur }\end{array}$ & $16,2 \%$ & $16,2 \%$ \\
\hline Ouvrier, personnel de service & $16,1 \%$ & $16,5 \%$ \\
\hline Autres, sans profession & $10,3 \%$ & $10,1 \%$ \\
\hline Total & $100 \%$ & $100 \%$ \\
\hline Effectifs & 195671 & 15612 \\
\hline
\end{tabular}

Champ : étudiants français seulement, hors indéterminés

Source : Un mythe à détruire?, op.cit, p. 211.

Ensuite concernant les non bacheliers, on observe que le cursus vincennois, qui mélange résolument bacheliers et non bacheliers, ne semble guère adapté, attendu que leur part passe de $46 \%$ en $1^{\text {er }}$ cycle à $13 \%$ en $3^{\text {e }}$ cycle. Au demeurant, ces non bacheliers ont un profil assez spécifique attendu que la majorité a le « niveau bac ». Ce qui, finalement, les rapproche beaucoup des étudiants ordinaires. Mais étant salariés en reprises d'études, travaillant souvent à plein temps, ils sont nettement plus âgés que les étudiants traditionnels et prennent aussi souvent plus de temps pour faire leurs études.

Tableau n 2 : L’origine scolaire des Vincennois selon le cycle en 1976-1977

\begin{tabular}{|l|c|c|c|c|}
\hline & $\mathbf{1}^{\mathbf{e r}}$ cycle & $\mathbf{2}^{\mathbf{e}}$ cycle & $3^{\mathbf{e}}$ cycle & Ensemble \\
\hline Bacheliers français & $29 \%$ & $43 \%$ & $46 \%$ & $35 \%$ \\
\hline Non-bacheliers français & $22 \%$ & $17 \%$ & $8 \%$ & $20 \%$ \\
\hline Bacheliers étrangers & $24 \%$ & $28 \%$ & $41 \%$ & $26 \%$ \\
\hline Non-bacheliers étrangers & $24 \%$ & $12 \%$ & $5 \%$ & $19 \%$ \\
\hline Total & $100 \%$ & $100 \%$ & $100 \%$ & $100 \%$ \\
\hline Effectifs & 17501 & 8953 & 1716 & 28170 \\
\hline
\end{tabular}

Source : Un mythe à détruire?, op.cit, p. 208. 
Enfin, analysant les professions exercées par ces salariés, on note que les ouvriers sont rares et que Vincennes répond déjà à une demande de formation continue, plutôt généraliste, émanant pour l'essentiel des professions intermédiaires, ou d'employés, provenant du secteur tertiaire et plus spécialement des secteurs éducatifs, sociaux, paramédicaux et artistiques, autant de secteurs particulièrement actifs en mai 68 comme après. Bref la «classe ouvrière » - objet à l'époque de tant de discours, fantasmes politiques - n'est pas vraiment présente en masse à Vincennes. Ou alors, il s'agit d'individus très atypiques, aux trajectoires heurtées, à l'héritage culturel, social, politique, religieux, etc., particulièrement complexe et souvent déjà lancés dans une démarche d'autodidaxie via le syndicalisme, la politique, etc. ${ }^{15}$.

En fait, et que ce soit au plan des caractéristiques sociales ou scolaires, tout se passe comme si c'est essentiellement aux marges du public universitaire habituel, ou si l'on veut de proche en proche, que Vincennes réussira à conquérir un nouveau public. Cette extension du public universitaire à d'autres populations, notamment plus âgées, touche d'ailleurs l'ensemble des universités françaises des années 70, même si c'est à un degré moindre. Et tout se passe comme si à l'époque, l'université française répond à une demande sociale forte, à un « désir d'apprendre » pour reprendre le titre d'un ouvrage de l'époque consacré à Vincennes ${ }^{16}$, caractéristique de toute une génération et notamment nourri par le flot des remises en cause, aspirations, interrogations collectives initiées en mai 68.

Voici par exemple comment Jean-François Le Ny, un professeur de psychologie expérimentale de Vincennes d'origine populaire et provinciale proche du Parti communiste français et qui fut aussi un des plus ardents participationnistes, décrit le public de cette université : «il y avait dans la région parisienne, à la fin des années 1960, plusieurs dizaines de milliers de jeunes hommes et femmes que les vicissitudes de l'existence avaient empêchés de passer le baccalauréat et de suivre des études universitaires, alors qu'ils en étaient intrinsèquement capables. Ils étaient très souvent, en cette période de plein emploi, déjà dotés d'une situation professionnelle, et ils y avaient généralement acquis un

\footnotetext{
${ }^{15} \mathrm{Cf}$. Claude Fosse-Polliak, « L'accès dérogatoire à l'enseignement supérieur, les autodidactes de SaintDenis », Revue française de sociologie, XXXII, 1991.

${ }^{16}$ Vincennes ou le désir d'apprendre, Jacqueline Brunet et ali, éditions Alain Moreau, 1979.
} 
niveau de compétence réelle qui dépassait sensiblement celui des diplômes qu'ils possédaient. C'est cette population, en fait très auto-sélectionnée, qui vint massivement à Vincennes, à la fois pour compléter ses connaissances de base, et pour acquérir des diplômes officiels, seuls moyens, dans le système français, d'avoir accès à de meilleurs postes et à des rémunérations plus élevées. Vincennes joua ainsi, durant une dizaine d'années, et en dépit de toutes les vicissitudes, un formidable rôle social et culturel de rattrapage universitaire et de formation de haut niveau pour adultes. [...] Mais peu à peu, me semble-t-il, ce "gisement" ou ce "stock", comme on dit vilainement, de capacités et de désirs sociaux diminua et finit pas s'épuiser. $)^{17}$

\section{Vincennes ou le triomphe de la pédagogie charismatique?}

Par son ouverture maximale, la diversification de ses filières et le caractère novateur de son enseignement, Vincennes saura donc répondre à une demande sociale, mais aussi intellectuelle, diffuse fortement liées à une conjoncture historique déterminée. Et il semble bien que la philosophie d'un Gilles Deleuze par exemple, dont certains localement souhaiteraient d'ailleurs faire l'icône de Paris 8 en rebaptisant de son nom cette université, offre une belle synthèse de l'esprit vincennois de l'époque.

Mais tout cela est très paradoxal. En effet d'un côté Vincennes a manifestement su répondre à une demande sociale, intellectuelle, forte, au point même de finir par devenir un mythe et être une des universités françaises les plus connues à l'étranger. Mais d'un autre, le bilan en matière de démocratisation universitaire paraît mitigé et les mécanismes de reproduction des inégalités sociales, scolaires semblent avoir fonctionné, ici comme ailleurs.

Pour comprendre ce paradoxe, il est nécessaire de s’intéresser au fonctionnement pédagogique de l'institution. Mais la tâche est malaisée. Déjà parce qu'il est difficile d'objectiver systématiquement les pratiques pédagogiques des enseignants, comme les

\footnotetext{
${ }^{17}$ Cf. « Autobiographie de Jean-François Le Ny », dans François Parot et Marc Richelle (dir.), Psychologues de langue française, autobiographies, Paris, PUF, 1992, p. 277-278.
} 
pratiques d'études des étudiants. Ainsi, les archives ne disent pas grand-chose à ce sujet et les témoignages ne sont pas très éclairants. De même, ces pratiques ont été très diverses d'un département à l'autre, comme d'un enseignant à l'autre. D'où la difficulté à appréhender la résultante de l'ensemble, le chercheur se trouvant alors placé devant une espèce de boîte noire. Laquelle renvoie d'ailleurs aux difficultés que nous rencontrons encore aujourd'hui pour objectiver nos propres pratiques. Ou dit autrement à notre inconscient pédagogique, qu'il soit individuel ou collectif.

Et de fait, il est particulièrement difficile d'avoir une idée claire de sa pratique comme des effets tant de son enseignement que de celui résultant du collectif enseignant. On dispose certes d'informations sur le nombre de diplômes délivrés, sur les programmes d'enseignement, échelles de notation, voire de travaux portant sur «les catégories de l'entendement professoral ", les copies des étudiants, etc., mais chacun ressent le manque cruel de médiations qui nous permettraient de mieux savoir ce que nous faisons quand nous enseignons et, pour l'essentiel, parlons... Bref notre pratique, et plus encore ses effets, nous sont encore très obscurs. Et c'est ce qui fait qu'assez curieusement travaillant sur l'histoire de Vincennes, nous n'avons pas tant eu l'impression de travailler sur du passé, que sur du présent. Et finalement de n'arriver à comprendre ce passé qu'en faisant un détour par notre propre expérience. Ce qui risque de conduire à une forme de cercle quelque peu anhistorique, puisque faisant l'impasse des spécificités historiques de l'époque.

Etudiant les pratiques pédagogiques vincennoises, on ne peut déjà qu'être frappé par la persistance du cours magistral, pourtant si vilipendé en mai 68 en raison notamment de son caractère autoritaire et élitiste. En effet, que voit-on quand on regarde les cours filmés de Deleuze, Châtelet, Lyotard ou d'autres ? Et bien un "grand professeur » qui parle dans une ambiance, souvent très enfumée, et des étudiants qui prennent des notes.

Alors certes, l'humeur politique du moment fait que certains auditeurs n'hésitent pas à interpeller, voire à admonester publiquement l'enseignant, de façon par exemple à lui rappeler la prééminence du politique, et donc des impératifs pratiques, moraux ou politiques, sur l'académique, l'intellectuel ou le pédagogique. Mais ce dispositif, somme 
toute très traditionnelle, semble avoir été très courant, notamment dans les cours des principaux ténors vincennois et plus généralement dans une discipline particulièrement livresque et symboliquement dominante comme la philosophie à Vincennes. Qui, pour cette raison d'ailleurs, donnera le ton tant idéologique, politique, que pédagogique, à nombre d'autres disciplines. Ce dont se désolera d'ailleurs Jean-Claude Passeron.

Par exemple, voici ce qu'en dit Michel Foucault en 1971 dans un entretien accordé à la Partisan Rewiew : "En France, on a violemment critiqué le système des conférences: le professeur arrive, reste derrière son bureau pendant une heure, dit ce qu'il a à dire, et l'étudiant n'a pas la possibilité de discuter. Les réformistes préfèrent la formule du séminaire, en ce qu'elle repose sur la liberté: le professeur cesse d'imposer ses idées et l'étudiant a le droit de parler. C'est vrai... Mais ne pensez-vous pas qu'un professeur qui prend la responsabilité d'étudiants au début de l'année, les fait travailler en petits groupes, les invite à entrer dans son propre travail, partage avec eux ses problèmes et ses méthodes, ne pensez-vous pas que, dans une telle formule, les étudiants sont encore plus déformés à la fin du séminaire que s'ils avaient simplement suivi une série de conférences? Ne vont-ils pas considérer comme acquis, naturel, évident et absolument vrai ce qui, après tout, n'est que le système, le code, la grille de leur professeur? Ne courent-ils pas le risque que le professeur leur impose ses idées de manière beaucoup plus insidieuse? Je ne veux pas défendre la conférence à tout prix, mais je me demande si elle n'a pas, à vrai dire, une sorte d'honnêteté brute, pourvu qu'elle précise ce qu'elle est: non pas la proclamation d'une vérité, mais la présentation, au stade expérimental, d'un travail qui a ses hypothèses, ses méthodes, et qui donc laisse le champ libre à la critique et aux objections : l'étudiant est libre de révéler ses maladresses. Naturellement, les séminaires et les groupes de travail sont nécessaires, mais plus, je crois, pour éprouver des méthodes que pour permettre l'exercice de la liberté.

Lorsque je fais une conférence un peu dogmatique, je me dis: je suis payé pour apporter aux étudiants une certaine forme et un certain contenu de savoir ; je dois fabriquer ma conférence ou mon cours un peu comme on fabriquerait une chaussure, ni plus, ni moins. Je conçois un objet, j'essaie de le fabriquer du mieux que je peux. Je me donne beaucoup de mal (pas toujours sans doute, mais souvent), j'apporte cet objet dans la salle de conférences, je le montre et, ensuite, je laisse le public libre d'en faire l'usage qui lui plaît. Je me considère 
plus comme un artisan fabricant un objet et l'offrant à la consommation que comme un maître faisant travailler ses esclaves. ${ }^{18}$

En fait, il semble avoir existé une certaine division du travail pédagogique, plus ou moins déniée, entre enseignants, les uns professant magistralement et usant donc d'une pédagogie de type charismatique finalement très traditionnelle, tandis que d'autres usent de dispositifs plus interactifs et sont plus tournés vers la pratique, soit les tâches ancillaires : notamment dans le cadre des cours d'initiation, d'apprentissages de base, de méthodologie, etc. Et manifestement cette division suit, grosso modo, l'échelle du prestige intellectuel ou politique, celle liée à l’ancienneté, au grade, au sexe, etc.

Alors dans les départements dont les cursus étaient les moins structurés formellement - et qui souvent étaient ceux où l'exigence d'égalité entre enseignants, mais aussi entre enseignés et enseignants, personnels administratifs et techniques, était la plus fortement revendiquée - cette division du travail n'est pas nécessairement formalisée dans une espèce d'organigramme a priori, ou une hiérarchie fonctionnelle clairement explicitée. Mais elle résulte plutôt de la position de chacun dans les hiérarchies symboliques, politiques et finalement sociales. Ainsi dans ces départements chacun semble, après quelques tâtonnements initiaux liés notamment à la nouveauté de l'expérience, avoir fini par trouver, pour parler comme Aristote, son « lieu naturel ». C'està-dire celui convenant le mieux à ce qu'il était socialement.

À Vincennes, il y aura donc loin entre le «petit prof » voué aux tâches pédagogiques ordinaires et qui, sur le modèle du prêtre membre d'une église bien hiérarchisée, se consacre pour l'essentiel aux tâches d'évangélisation et d'élévation de laïcs plein de bonne volonté scolaire, souvent plus jeunes, plus féminins, plus français et plus bacheliers que la moyenne et en phase d'accumulation d'un capital scolaire éventuellement monnayable sur le marché national des concours de recrutement ${ }^{19}$ -

\footnotetext{
18 "Conversation avec Michel Foucault », texte reproduit dans Dits et écrit 1954-1988, II, 1970-1975, Gallimard, 1994, p. 190-191. Cette apologie du cours magistral se retrouve aussi chez Deleuze. Et elle s'accompagne aussi chez lui de toute une critique de la « discussion » en cours. Sur ce point, voir ce qu'en dit ce philosophe quand il parle du «Professeur ». Cf. L’Abécédaire de Gilles Deleuze, film réalisé par Pierre-André Boutang, éditions du Montparnasse, 2004.

19 Type étudiant manifestement plus répandu en histoire, littérature française, géographie, langues... Lesquelles disciplines ont, et conservent, un public plus «scolaire » que les autres (et travaillant plus
} 
lesquelles tâches entrent souvent en écho avec ses convictions pédagogiques et démocratiques les plus profondes -, le chargé de cours entré sur critères politiques travaillant et discutant sur des sujets directement politiques faisant l'objet de sa thèse (souvent d'inspiration maoïste en début de période) avec des étudiants plus âgés que la moyenne, eux-mêmes très politisés (ou qu'il s'agit alors de politiser afin d'en faire de futurs militants révolutionnaires) et a priori moins préoccupés par la question des débouchés professionnels ${ }^{20}$, le chargé de cours artiste professionnel, ou critique d'arts, en lutte contre l'intellectualisme ordinaire, et sans cesse renaissant, de l'université et en voie de reclassement social (comme le dit l'un d'eux, critique aux Cahiers du cinéma, en matière de goût " la critique a un privilège sur l'université ") ${ }^{21}$, ou encore le chargé de cours d'une discipline nouvelle comme les sciences de l'éducation adepte de la «dynamique de groupe », du «potentiel humain » ou de la «bioénergie » répondant aux demandes d'expérimentation pédagogique, "développement personnel », voire thérapeutiques, d'inspiration plus ou moins freudienne ou rousseauiste émanant de salarié(e)s provenant du secteur éducatif, social ou paramédical. Et pour finir - mais cette typologie sommaire mériterait d'être développée -, le virtuose, ou «grand intellectuel » parisien (le plus souvent philosophe et généralement doté des titres scolaires les plus canoniques) déjà connu avant d'arriver à Vincennes, aux dispositions plus ou moins critiques et prophétiques et capable, en mobilisant les concepts hérités de la tradition et / ou «fabriqués » pour l'occasion (ainsi, Gilles Deleuze parlera de la philosophie comme d'un lieu de "fabrication de concepts » et du plaisir qu'il y a, dans cette discipline, à surmonter «la censure » qu'exerce habituellement l'histoire de la philosophie pour arriver, enfin, à parler « en son propre nom ») $)^{22}$, d'opérer ce travail de retraduction, universalisation des

souvent dans l'Éducation nationale) et entretiennent dès l'origine un lien plus étroit avec les concours de recrutement de l'enseignement secondaire.

${ }^{20}$ Ce type d'étudiant paraît avoir été plus courant en sociologie, science politique, économie, disciplines au recrutement très masculin, dont les objets sont plus directement politiques, où le taux d'étudiants étrangers est plus élevé et le droit d’entrée semble avoir été plus faible, tant du côté étudiant qu'enseignant, les disciplines lettrées traditionnelles continuant souvent à être implicitement régulées par les concours de l'enseignement secondaire.

${ }^{21}$ Cette opposition se retrouve aussi en partie en littérature française comme en philosophie, où à la figure du « simple prof » s'oppose celle de «l'écrivain », du " poète » ou du « philosophe », et plus largement du "créateur ». Et elle recoupe aussi celle, très hiérarchisante, du "pédagogue » et du " chercheur », et particulièrement prégnante dans l'enseignement supérieur.

${ }^{22}$ C. Soulié, « Le destin d'une institution d'avant-garde : histoire du département de philosophie de Paris 
préoccupations profanes d'un public excédant largement les bornes de celui de l'université traditionnelle. En eux résonne l'esprit du lieu qu'ils sauront interpréter, mettre en forme, sans nécessairement s’y aliéner.

\section{Marché libre et concurrence charismatique généralisée}

Nous avons souligné la diversité des pratiques pédagogiques en fonction du statut, genre, discipline, volume et formes de capitaux, etc., des enseignants. Mais cellesci sont aussi déterminées en amont par la structure formelle des cursus d'enseignements, elle-même résultant d'un certain état du rapport de forces/collaboration entre enseignants et étudiants, comme entre enseignants de rangs différents. Et de fait, si dès le départ dans de gros départements comme l’anglais ou la psychologie (ce qui, de facto, leur imposera des contraintes de gestion afin de réguler a minima des flux importants d'étudiants, comme d'enseignants) ${ }^{23}$ les étudiants doivent suivre un cursus très rigide avec un système de cours obligatoires et hiérarchisés en fonction notamment de l'année d'études et donc de leur «niveau », dans d'autres ils sont intégralement libres de choisir eux-mêmes les cours composant leur diplôme.

Tel est par exemple le cas en philosophie, sociologie, arts, psychanalyse, littérature française, etc. D’où la critique par Deleuze, Châtelet et d'autres du thème de la nécessaire « progressivité » des études et leur éloge - paradoxal du point de vue du sens commun pédagogique - de l'extrême disparité du public vincennois ${ }^{24}$. Public dont Deleuze fera d'ailleurs souvent l'éloge, en en parlant comme de « la splendeur de Vincennes ».

On retrouve alors, entre autres, les effets d'un a priori éthicopolitique extrêmement puissant à l'époque et postulant l'égalité potentielle de chacun devant le

\footnotetext{
VIII », Histoire de l'éducation, n’77, 1998, p. 62.

${ }^{23}$ Sachant que le département d'anglo-américain aura aussi à gérer une bonne partie de la masse des étudiants d'autres disciplines devant obligatoirement faire un enseignement de langue pour valider leur licence.

${ }^{24}$ Sur ce point : François Châtelet, « Disparité et non hiérarchie », in Vincennes ou le désir d'apprendre, op.cit, p. 126 et suivantes.
} 
savoir et qui, en conséquence, en appelle à l'autogestion par chaque étudiant de sa propre formation. Ou dit autrement, c'est l'idée que chacun est le mieux placé pour savoir ce dont il a besoin, où est son « désir », pour identifier ce qui «lui parle », «l'interpelle subjectivement ». Et donc avec quel enseignant il peut se produire une «rencontre » de type plus ou moins charismatique et analogue à celle qui s'opère entre un maître et ses disciples ${ }^{25}$. Et une institution universitaire « ouverte à tous » comme Vincennes se doit d'y répondre, en offrant notamment la palette d'enseignements la plus diversifiée qui soit, et la moins contraignante possible. Car cette diversité, souplesse extrêmes favorisent, pour reprendre encore le vocabulaire deleuzien, la «multiplicité » des «branchements » potentiels possibles entre enseignants et enseignés. Sachant que cette période intellectuelle et historique se caractérise aussi, après l'essoufflement d'un marxisme léninisme désormais perçu comme «autoritaire» et bientôt critiqué pour son «totalitarisme» par les «nouveaux philosophes ${ }^{26}$, par un épanouissement de la «philosophie du désir». Philosophie dont Deleuze sera d'ailleurs un des grands promoteurs, tout en en critiquant âprement la version psychanalytique et lacanienne ${ }^{27}$.

Cette absence de structuration formelle du cursus s'accompagne aussi souvent d'une coordination pédagogique minimale entre enseignants. Ainsi, et toujours en philosophie, les réunions pédagogiques sont inexistantes (la pédagogie étant d'ailleurs traditionnellement méprisée par les philosophes, ce que souligne bien une formule courante dans ce milieu et selon laquelle: "La philosophie est à elle-même sa propre pédagogie. »), les enjeux politico-intellectuels passant au premier plan. Pourtant au début, Michel Foucault aurait bien voulu « tenir en main » son département. Mais la conjoncture politique du moment, comme le type de recrutement qu'il avait lui-même opéré, ne lui en donneront guère les moyens. Et finalement, il sera quelque peu dépassé par sa créature, tout en se nourrissant intellectuellement et politiquement de l'expérience de toute cette génération. Du reste, il ne s'attardera guère à Vincennes, supportant mal de se voir

\footnotetext{
${ }^{25}$ Concernant l'importance du thème de la «rencontre » dans la pédagogie deleuzienne: Sébastien Charbonnier, Deleuze pédagogue, la fonction transcendantale de l'apprentissage et du problème, L'Harmattan, 2009.

${ }^{26}$ Dont l'un d'entre eux (André Glucksmann) enseignera d'ailleurs momentanément à Vincennes et passera donc du maoïsme le plus radical à la nouvelle philosophie.

${ }^{27}$ Cf. L’Anti-CEdipe, avec Félix Guattari, Minuit, 1972.
} 
interpellé publiquement lors des cours. Et, dès 1970, il rejoint le Collège de France, abandonnant alors le soin de la gestion du département de philosophie à François Châtelet.

La situation était relativement proche en sociologie, sachant que cette discipline occupait une position symboliquement dominée par la philosophie ${ }^{28}$. Si au début l'équipe initiale rassemblée par Passeron arrive à mettre sur pied un programme d'enseignement relativement classique avec un minimum de progressivité dans les enseignements, très rapidement, et sous l'effet notamment de la pression des intérêts, demandes de biens de salut politiques des étudiants comme des enseignants les plus militants, le cursus se libéralise et les programmes d'enseignements se transforment. Ainsi, une sociologie politique d'inspiration marxiste, et plus ou moins scolastique en raison notamment de l'empreinte althussérienne initiale comme de l'absence de formation préalable à la recherche empirique des enseignants recrutés, prend largement le dessus. Les cours de statistiques, suspects d'être des outils de la pensée d'Etat, disparaissent complètement, ceux de méthodologie (observation, entretien, questionnaire) sont quasiment absents ou remplacés par une « recherche action » à visée d'abord militante et souvent d'inspiration maoïste au début (ou comme le disait le président Mao, fort prisé à l'époque: «Qui n’a pas fait d'enquête n'a pas droit à la parole »), et les contraintes en matière de cursus disparaissent complètement. En 1971, chaque étudiant compose intégralement son cursus. Le contenu précis de chaque licence, et donc la formation reçue, varie alors considérablement d'un étudiant à l'autre.

\footnotetext{
${ }^{28}$ Ce développement s'inspire librement de : «La sociologie à Vincennes : une discipline dispersée », dans Un mythe à détruire?, op.cit, p. 315 et suivantes.
} 
Programme du département de sociologie de Vincennes en 1971-1972

\begin{tabular}{|c|c|}
\hline 1. CLASSE OUVRIÈRE & \\
\hline $\begin{array}{l}\text { Processus d'implantation du PCF à travers l'étude critique de l'origine du PC } \\
\text { (82) }\end{array}$ & J. Trat \\
\hline L'intégration des syndicats comme appareil d'État (problème de la participation) (173) & C. Weckerlé \\
\hline Division et unité de la classe ouvrière (immigrés, taille des entreprises, secteurs de pointe) (32) & F. Duroux \\
\hline $\begin{array}{l}\text { Le problème des alliances : classe ouvrière, paysannerie et classes moyennes } \\
\text { (Unité de la classe ouvrière et unité populaire) (132) }\end{array}$ & C. Weckerlé \\
\hline Les partis et les syndicats en Italie (35) & I.Séminatore \\
\hline La conscience de classe : du tiercé à la révolution (281) & M. Joubert \\
\hline Mouvement ouvrier et mouvement socialiste en France (50) & $\begin{array}{l}\text { B. Conein, M.-N. } \\
\text { Thibault, Fr. Duroux }\end{array}$ \\
\hline 2. IMPERIALISME ET SOUS-DÉVELOPPEMENT & \\
\hline Structure des classes dans les formations dominées de l'Amérique latine (44) & Y. Duroux \\
\hline Structure des classes dans les formations dominées de l'Afrique noire (118) & P.-P. Rey \\
\hline $\begin{array}{l}\text { Structure des classes dans les formations dominées du Moyen-Orient, Maghreb, pays } \\
\text { méditerranéens }\end{array}$ & \\
\hline Algérie, paysannerie et émigration (129) & J.-P. Briand \\
\hline Égypte (22) & M. Benzine \\
\hline Grèce (55) & C. Tsoucalas \\
\hline Structures des classes dans les formations dominées de l'Asie du Sud-Est (47) & $\begin{array}{l}\text { Y. Duroux, S. Lazarus, } \\
\text { G.Althabe, C. Mallet, } \\
\text { Ravoajanahary } \\
\end{array}$ \\
\hline $\begin{array}{l}\text { Types d'intervention impérialiste (pays capitalistes et pays socialistes) - Coopération culturelle, } \\
\text { présence diplomatique, intervention militaire, investissements: }\end{array}$ & \\
\hline Rôle de l'armée dans la lutte des classes (84) & S. Lazarus, J.-M. Faure \\
\hline Forme de l'État dans les pays dominés par l'impérialisme & M. Benzine \\
\hline Sociétés agraires et sous-développement (58) & G. Leclerc \\
\hline 3. PROBLĖMES DU SOCIALISME & \\
\hline Les transformations de l'agriculture chinoise (95) & J.-M. Faure \\
\hline Les rapports villes-campagnes (1928-1934) en URSS & J.-M. Faure \\
\hline Le débat organisationnel (70) & M. Grumbach \\
\hline Les rapports de travail et les syndicats soviétiques (39) & M. Grumbach \\
\hline Le socialisme et la question des femmes & C. Dufrancatel \\
\hline Analyse comparée de la formation de l'État soviétique et de l'État chinois (115) & S. Lazarus \\
\hline La politique extérieure de la République populaire de Chine (120) & C. Mallet \\
\hline L’État socialiste et la guerre (81) & S. Lazarus \\
\hline La gestion des entreprises en URSS (18) & A.Bory \\
\hline 4. PROBLÈMES ACTUELS DE L'ÉTAT & \\
\hline Hégémonie et lutte des classes (53) & B. Conein, Y. Duroux \\
\hline Classes sociales et partis politiques (problèmes de représentation et d'organisation) (81) & M. Loewi, N. Poulantzas \\
\hline L'État socialiste et la guerre (cf. grappe problèmes du socialisme) & S. Lazarus \\
\hline Formes actuelles de l'État aux USA (52) & C.Tsoucalas \\
\hline Les partis et les syndicats en Italie (cf. grappe ouvrière) & I. Seminatore \\
\hline Dictature culturelle et mécanisme de l'idéologie dans les pays impérialistes (69) & J.-P. Dollé \\
\hline Problème de l'intégration des syndicats (cf. grappe ouvrière) & C. Weckerlé \\
\hline $\begin{array}{l}\text { Formes de l'État dans les pays dominés par l'impérialisme (cf. grappe } \\
\text { impérialisme et sous-développement) }\end{array}$ & M. Benzine \\
\hline 5. PAYSANNERIE ET PETITE BOURGEOISIE & \\
\hline Les classes sociales à la campagne dans les métropoles impérialistes (15) & J.-P. Grossein \\
\hline Le problème des alliances : paysannerie et classes moyennes (cf. La classe ouvrière) & C. Weckerlé \\
\hline La bourgeoisie du XIII ${ }^{\mathrm{e}}$ au $\mathrm{XX}^{\mathrm{e}}$ siècle & M. Meyer, P. Sorlin \\
\hline
\end{tabular}




\begin{tabular}{|c|c|}
\hline Élites et culture dans les formations dominées (86) & M. Meyer \\
\hline Types d'intellectuels 1. La presse et les journalistes (65) & $\begin{array}{l}\text { J.-C. Passeron, H. Peretz, A. } \\
\text { Gras }\end{array}$ \\
\hline \multicolumn{2}{|l|}{ 6. APPAREILS ET CONTRÔLES } \\
\hline \multicolumn{2}{|l|}{ L'école } \\
\hline Les idéologies pédagogiques (142) & B. Conein, P. de Gaudemar \\
\hline Scolarisation et société de classe (120) & J.-M. Chapoulie, P. de Gaudemar \\
\hline École et politique (48) & J.-M. Chapoulie \\
\hline École et marché du travail ( $2^{\mathrm{e}}$ semestre) & J.-M. Chapoulie \\
\hline Pédagogie, moyens d'expression et politique (249) & M. Joubert \\
\hline L'opinion politique - critique de la politique des enquêtes de sondages (26) & C. Dufrancatel, Darbre \\
\hline \multicolumn{2}{|l|}{ Institutions pénitentiaires, psychiatriques et para-psychiatriques } \\
\hline Cycles pénitentiaires et cycles psychiatriques (41) & R. Castel, J. Donzelot \\
\hline Fonctionnement de la pratique psychiatrique (21) & E.Wulff \\
\hline Observation ethnométhodologique du procès (66) & N. Herpin \\
\hline Observation ethnométhodologique du procès (22) & M. Naffrechoux \\
\hline Police, justice, prison (137) & D. Defert \\
\hline L'argent dans la pratique et dans la théorie psychanalytique ( $2^{\mathrm{e}}$ semestre) & J.-P. Grossein \\
\hline Processus de contrôle des faits psychiatriques et criminologiques (138) & N. Herpin, R. Castel \\
\hline L'enfance inadaptée (20) & C. Wagman \\
\hline La famille (100) & $\begin{array}{l}\text { C. Dufrancatel, F. } \\
\text { Duroux }\end{array}$ \\
\hline Observation ethnographique des pratiques familiales ( $2^{\mathrm{e}}$ semestre) & N. Herpin \\
\hline \multicolumn{2}{|l|}{ 7. ARTS ET URBANISME } \\
\hline Objet d'une sociologie urbaine (107) & M. Bénard \\
\hline Exode rural et urbanisme (43) & M. Bénard \\
\hline Loisir et urbanisme (6) & M. Bénard, J.-P. Martinon \\
\hline Méthodologie pour des enquêtes sur les spéculations et les expulsions (3 gpes) (112) & D. Defert \\
\hline Objet d'une sociologie de l'Art (38) & R. Lelièvre, H. Peretz \\
\hline Introduction à la sociologie et à l'économie du cinéma (56) & M. Jakubowicz \\
\hline Mythe et littérature (64) & J.-P. Martinon \\
\hline Les monopoles de la production des normes esthétiques (22) & H. Peretz \\
\hline Pratiques religieuses et pratiques artistiques (54) & R. Lelièvre \\
\hline Fonctions du discours d'accompagnement & J.-P. Martinon \\
\hline \multicolumn{2}{|l|}{ 8. UV INTERDISCIPLINAIRES } \\
\hline La bourgeoisie du XIII ${ }^{\mathrm{e}}$ au XX $\mathrm{X}^{\mathrm{e}}$ & M. Meyer, P. Sorlin \\
\hline Mouvement ouvrier et mouvement socialiste en France (la Commune et 36) & $\begin{array}{l}\text { B. Conein, M.-N. Thibault, } \\
\text { Fr. Duroux }\end{array}$ \\
\hline Mythe et littérature & J.-P. Martinon \\
\hline Le racisme & $\begin{array}{l}\text { C. Dufrancatel, R. } \\
\text { Galissot, M. Rebérioux }\end{array}$ \\
\hline Nationalisme et question nationale & $\begin{array}{l}\text { R. Gallissot, M. Loewy, } \\
\text { N. Poulantzas }\end{array}$ \\
\hline Sémiologie : introduction (92) & L. Prieto \\
\hline Sémiologie : séminaire sur la communication et la connaissance (82) & L. Prieto \\
\hline L’image de la femme dans la littérature contemporaine (41) & $\begin{array}{l}\text { C. Dufrancatel, } \\
\text { G. Rosowsky, J. Stora, B. } \\
\text { Slama }\end{array}$ \\
\hline $\begin{array}{l}\text { Atelier de traduction (allemand), texte sur l'argent dans la psychanalyse, la } \\
\text { famille }\end{array}$ & J.-P. Grossein \\
\hline
\end{tabular}

Source : Un Mythe à détruire ?, op.cit, p. 338.

Le chiffre figurant entre parenthèses après chaque titre de cours correspond au nombre d'UV délivrées aux $1^{\text {er }}$ et $2^{\mathrm{e}}$ semestres (hors additifs). Il donne une idée du « succès » de chaque cours auprès des étudiants. 
Comme l'explique J.-C. Passeron : «Alors donc, finalement, j'avais quand même mis en place un département. Et donc bien sûr, je ne voulais pas être élu le président ou chef, en pleine crise maoïste... Où les petits chefs, je ne sais pas... C'était un collectif où tout le monde votait, mais où ils admettaient dans leur intérêt bien compris (avoir de plus en plus, être plus ou moins titularisés, etc.), qu'il valait mieux que je sois le représentant extérieur du département. Mais à l'intérieur normalement, j'avais pas plus de droit que les autres dans la fabrication des emplois du temps... C'était un chaos de conflits entre les tendances politiques gauchistes, qui étaient souvent des jeunes étudiants n'ayant pas vraiment fini leurs études, qu'on prenait comme chargés de cours. » La position décrite par J.-C. Passeron est peu ou prou partagée par l'ensemble des enseignants vincennois qui, plus âgés et dotés d'un certain capital et/ou occupant des fonctions de responsabilité, servent de caution (intellectuelle, politique, gestionnaire) au collectif vincennois, tant vis-à-vis de l'extérieur du département, que de l'université dans son ensemble, notamment dans ses rapports avec la presse, le ministère, etc. Et ce chaos n'est guère propice au développement d'une "pédagogie rationnelle » collectivement concertée, pourtant appelée de leurs vœux par les auteurs des Héritiers. Ainsi, Robert Castel tire un bilan mitigé de l'expérience : « Je crois qu'au début, il y a dû avoir une entrée à Vincennes de gens qui étaient de milieux vraiment populaires, qui ont cru à cette chose à laquelle on croyait aussi un peu. [...] Et là au début, beaucoup ont facilement compris que ce n'était pas, que ce n'était sans doute pas possible. [...] L'idéal un peu démocratique qui était là au début, bon ça ne s'est pas réalisé. Bon par contre, ça a servi à des gens qui étaient déjà, qui n'étaient pas complètement disons de milieux populaires, sauf exceptionnellement. C'était souvent des gens qui avaient une trajectoire un peu universitaire, mais ça avait foiré, ou bien ils n'avaient pas continué. Eux, ça les a servis. ${ }^{29}$

Finalement, une des principales innovations pédagogiques vincennoises résulte dans l'invention d'une sorte de marché libre académique sur lequel chaque enseignant était placé en concurrence charismatique avec tous les autres dans la captation de la clientèle étudiante, situation propice aussi à tous les débordements démagogiques, ou clientélistes. Ainsi, la remise en cause du rapport de domination pédagogique traditionnel

\footnotetext{
${ }^{29}$ Entretien avec R. Castel réalisé le 6 décembre 2007.
} 
aboutira à la constitution d'un marché libre où chaque étudiant est censé savoir maximiser son profit, qu'il soit intellectuel, politique, académique ou autre. Résultat pour le moins paradoxal s'agissant d'une institution aussi gauchisante que Vincennes et qui, d'une certaine manière, traduit sans doute bien les ambigüités, ou du moins la complexité, de l'héritage politique de l'époque, certains gauchistes faisant d'ailleurs ensuite une belle carrière dans la presse, ou devenant des hommes d'affaire avisés.

\section{Classes sociales d'origine, trajectoires et orientations pédagogiques}

Concernant la démocratisation de l'accès au savoir, le bilan de Vincennes est donc plutôt mitigé. Et ce en raison notamment de ses « choix » pédagogiques particulièrement «libéraux» qui, par exemple, ne faciliteront guère l'orientation des étudiants non bacheliers les plus éloignés de la culture légitime, qui dans nombre de disciplines seront en quelque sorte livrés à eux-mêmes face au maquis des cours proposés. Choix entre guillemets, car résultant pour l'essentiel des circonstances et rapports de forces politiques du moment. Et c'est sans doute pour cette raison que Jean-Claude Passeron dira de cette institution que c'était « un univers a pédagogique » et qu'il tire aussi un bilan quelque peu critique de cette expérience : « enfin je voulais voir qu'est-ce qui pouvait sortir pédagogiquement d'une telle situation. Et donc, sans que ce soit une déconvenue, j'ai touché du doigt l'impossibilité de pratiquer à Vincennes un redépart pédagogique, enfin pour les étudiants. [...] La seule chose, c'est que le désordre ne fabrique jamais le réformisme. Si vous voulez, la première année il y a eu une bonne volonté, y compris réformiste, c'était le langage que l'on parlait. Quand on se réunissait - parce qu'il y avait des commissions à Vincennes. Par exemple, il y avait la commission pour les Unités de Valeur - comment fera-ton pour les examens? Il y avait le représentant de... Pour l'histoire, c'était presque toujours Madeleine Rebérioux, qui était un peu sur la même position que la mienne : c'est-à-dire de tirer quelque chose, même de cette atmosphère révolutionnaire, d'en tirer quelque chose. 
Comme moi. Et il y avait des représentants de tous les départements, qui la plupart étaient réformistes. Mais en philo, ils ne venaient pas... ${ }^{30}$

A contrario, il est évident que l'expérience de Vincennes répondra à une demande sociale forte, ou pour utiliser le vocabulaire deleuzien à un «désir » particulièrement puissant. Demande largement déterminée par la conjoncture sociale, politique et culturelle du moment et plus largement par les effets de long terme de la crise de 68 sur la société française. Demande dont on peut aussi se demander si elle ne sera pas spécialement portée par certaines classes, ou fractions de classes. Et ici, nous pensons notamment aux analyses qu'en 1979 Pierre Bourdieu développe dans La Distinction à propos des membres de la « petite bourgeoisie nouvelle » qui, comme il l'écrit à l'époque, ont partie liée avec «l'éducation permanente », entretiennent « une relation ambivalente au système scolaire » les portant notamment «à se sentir complices de toute espèce de contestation symbolique ». Lesquels feront donc alliance avec l'avant-garde académique du moment en lutte contre l'ancienne Sorbonne.

Dans le cas de Vincennes, il semble bien qu'autour des questions de pédagogie, et notamment de formalisation des cursus, degré de contrainte imposé aux étudiants, etc., on ait assisté à une lutte entre groupes sociaux antagoniques aux intérêts plus ou moins divergents et donc à une forme de lutte des classes plus ou moins déniées. Ainsi, il semble bien que les options pédagogiques les plus libérales et charismatiques aient été préférentiellement portées par les enseignants et étudiants d'origine sociale plus élevée (voire déclassés vers le bas), tandis que ceux d'origine populaire, ou en phase d'ascension sociale, défendront souvent des options plus scolaires et réglées, manifestement mieux en phase avec les impératifs de l'accumulation d'un capital scolaire nécessaire à leur projet d'ascension sociale ${ }^{31}$. Laquelle opposition recoupe aussi en partie des oppositions politiques plus générales (entre «anti participationnistes» et «participationnistes», «révolutionnaires » et «réformistes »). Ce qui pose alors la question de la définition

\footnotetext{
${ }^{30}$ Un mythe à détruire, op.cit. p.223.

31 "Faut-il rappeler que, dans l'histoire des utopies pédagogiques, l'idéal d'un enseignement sans institution ni sanction, dégagé des routines et des contrôles plébéiens des "hommes de métier » et visant à réveiller chez l'élève des dons ou des vérités qu'il possède déjà, ont presque toujours coïncidé avec une représentation aristocratique des disciples dignes d'y satisfaire?" Cf. Jean-Claude Passeron, La Réforme de l'université, op.cit, p. 214.
} 
pratique de la « pédagogie rationnelle » appelée de leurs vœux par Bourdieu et Passeron, comme des forces sociales et politiques susceptibles de porter un tel projet pédagogique.

\section{Références bibliographies}

BOURDIEU, Pierre e DARBEL, Alain. L'Amour de l'art. Paris: Éditions de Minuit, 1966. BOURDIEU, Pierre. Homo academicus. Paris: Éditions de Minuit, 1984.

BOURDIEU, Pierre; PASSERON, Jean-Claude. La reproduction, éléments pour une théorie du système d'enseignement. Paris: Éditions de Minuit, 1970.

BOURDIEU, Pierre; PASSERON, Jean-Claude. Les héritiers: Les étudiants et la culture. Paris: Éditions de Minuit, 1964.

BRUNET, Jacqueline et al. Vincennes ou le désir d'apprendre. Paris: Éditions Alain Moreau, 1979.

BUÉ, Alain. Josué de Castro, un visionnaire brésilien à Vincennes: Mário Soares, “Exilé en cravate". In DJIAN, Jean-Michel. Vincennes, une aventure de la pensée critique. Paris: Flammarion, 2009, p. 126-129.

CASTEL, Robert. e PASSERON, J.-C. Éducation, développement et démocratie. Paris-La Haye: Mouton, 1967.

CHARBONNIER, Sébastien. Deleuze pédagogue, la fonction transcendantale de l'apprentissage et du problème. Paris: L'Harmattan, 2009.

CHÂTELET, François. Disparité et non hiérarchie". BRUNET, Jacqueline et al. Vincennes ou le désir d'apprendre. Paris: Éditions Alain Moreau, 1979, p. 126 e seguintes.

DELEUZE, Gilles e GUATARRI, Félix. L'Anti-CEdipe. Capitalisme et schizophrénie. Paris: Éditions de Minuit, 1972.

DELEUZE, Gilles. L'Abécédaire de Gilles Deleuze. In BOUTANG, Pierre-André (Dir.). Paris: Éditions du Montparnasse, 2004 (filme).

FAURE, Edgar. Philosophie d'une réforme. Paris: Plon, 1969.

FOSSE-POLLIAK, Claude. L'accès dérogatoire à l'enseignement supérieur, les autodidactes de Saint-Denis. Revue française de sociologie, XXXII, 1991.

FOUCAULT, Michel. Dits et écrit 1954-1988, II, 1970-1975. Paris: Gallimard, 1994, p. 190-191. 
GÉRALD, Antoine e PASSERON, Jean-Claude. La Réforme de l'université. Paris: CalmannLévy, 1966.

LE GALL, Brice e SOULIÉ, Charles. Voyage en terre d'asile académique: éléments pour une histoire sociale des étudiants étrangers de Paris VIII et d'ailleurs. In SOULIÉ, Charles (Dir.). Un mythe à détruire? Origenes et destin du Centre universitaire expérimental de Vincennes. Saint-Denis: Presses Universitaire de Vincennes, 2012, p. 393-422.

LEGOIS, Jean-Philippe. Jeux d'échelle entre les premiers pouvoirs universitaires vincennois, 1968-1971. In SOULIÉ, Charles (Dir.). Un mythe à détruire? Origenes et destin du Centre universitaire expérimental de Vincennes. Saint-Denis: Presses Universitaire de Vincennes, 2012, p. 259-277.

PAROT, Françoise e RICHELLE, Marc Autobiographie de Jean-François Le Ny. In PAROT, Françoise e RICHELLE, Marc. (Dir.). Psychologues de langue française, autobiographies, Paris: Presses Universitaire de France, 1992, p. 277-278.

PASSERON, Jean-Claude. 1950-1980: I'université mise à la question: changement de décor ou changement de cap? In VERGER, Jacques (dir.). Histoire des universités en France.

Toulouse: Privat, 1986.

POULY, Marie-Pierre. L'anglais de la Sorbonne à Vincennes. In SOULIÉ, Charles (Dir.). Un mythe à détruire? Origenes et destin du Centre universitaire expérimental de Vincennes. Saint-Denis: Presses Universitaire de Vincennes, 2012, p. 282-313.

PROST, Antoine. Histoire générale de l'éducation. Paris: Perrin, vol. IV, 2004.

SOULIÉ, Charles. Le destin d'une institution d'avant-garde: histoire du département de philosophie de Paris VIII. Histoire de l'éducation, $n^{\circ} 77,1998$, p. 62, disponível: http://www2.univ-paris8.fr/sociologie/fichiers/soulie1998a.pdf

SOULIÉ, Charles. Un mythe à détruire? Origenes et destin du Centre universitaire expérimental de Vincennes (Dir.). Saint-Denis: Presses Universitaire de Vincennes, 2012.

Recebido em: 20/08/2013 Aprovado em: 10/02/2014

Universidade do Estado de Santa Catarina - UDESC Programa de Pós-Graduação em Educação - PPGE Revista Linhas Volume 15 - Número 29 - Ano 2014 revistalinhas@gmail.com 